\title{
From Neurology and Psychiatry to NeuroPsychiatry
}

\author{
Uriel Halbreich* \\ Director, Bio-Behavioral Research, Professor of Psychiatry, SUNY-AB, Buffalo, NY, USA \\ *Corresponding Author: Uriel Halbreich, Director, Bio-Behavioral Research, Professor of Psychiatry, SUNY-AB, Buffalo, NY, USA.
}

Received: July 02, 2019; Published: July 19, 2019

DOI: 10.31080/ASNE.2019.02.0085

One of my early Professors of Psychiatry apologetically joked:" Neurologists make very accurate diagnosis but cannot do much about it, we Psychiatrists may come up with a vague diagnosis or some symptomatic description but we are better at treatment". During my residency- rotation in Neurology, my mentor, the Professor and Chair stationed me at the office next to his and told his patients who suffered of headache or some other pains "Go to Dr Halbreich he will help you". I treated them quite successfully with hypnosis. Everybody was happy, including the Hospital`s Private Practice office who charged for "Professor supervised Patientcare".

As a resident in the Department of Neurology it was apparent that Neurologists in the General University Hospital were a very appreciated Medical Specialty, however, the Psychiatrists were perceived as "strange birds", they did not speak the same language as Internists and Surgeons, their practices were awkward and they did not mingle well with the other MDs. They actually suffered of the same stigma as their patients and even amplified it. The perception of Psychiatrists as second degree citizens in the Medical community was explicitly manifested to me during my internship. When I told my supervisor in surgery that I consider residency in Psychiatry, his response was "Uri, are you crazy you can be a very good Surgeon". He was not convinced when I attempted to explain that Psychiatry is on the verge of being the fastest developing field, Brain and the human mind are the most fascinating realm of medicine and I wish to be part of it. He summarized the chat:" $\mathrm{OK}$, so be a Neurosurgeon but now hurry to wash hand for Mrs. X appendectomy".

Recently, an important component of the improvement in the knowledge foundation of Psychiatry is the better delineation of physiology and biochemistry of the brain as well as continuous substantial development of dynamic Neuroimaging technologies. It has been increasingly apparent that the Central Nervous System (CNS) -the brain is the organ of cognition, emotions and coordination with the whole body and the environment. Current knowledge emphasizes the overlap between the two clinical disciplines that focus on the CNS - Neurology and Psychiatry. Indeed, one may argue that Psychiatry includes Psychological, Social, spiritual and cultural aspects while Neurology encompasses peripheral Neuromuscular functions as well as vascular abnormalities. So the overlap is not $100 \%$ but it is getting closer.

In practice, referral to a Psychiatrist or a Neurologist is often times according to patient's or Primary Care Physician (PMP) perception of complaints and not necessarily based on presumed underlying mechanisms. Initial referral may determine treatment roadmap, many Psychiatrists would treat patient`s clinical complaints without tests of potential patho-bio-physiology. Many Neurologists would perform neurological exam and -if no localized signs are demonstrated, would refer the patient to a Psychiatrist as a mental case.

How to rectify current dichotomy is a complex issue that is beyond a brief statement of need. First step is the recognition of intertwined association of all functions of the CNS. The pursuit of positive emotions, especially Happiness is an integral part of any Neurological intervention [1]. Integration of Psychiatry and Neurology into well-rounded NeuroPsychiatry is an absolute necessity for improvement of Patients` well-being. It starts with amendment of attitudes, education of trainees and the public as well as leaders of professional associations. Efforts should lead to establishment of functional partnerships on the way to integration and enhancement of well-being for all [2]. 


\section{Bibliography}

1. Halbreich U. "Pursuit of Happiness, Prosperity and Health (PHPH)". International Journal of Social Psychiatry 64.4 (2018): 307-308.

2. Halbreich U., et al. "Partnerships for Interdisciplinary Collaborative Global Well-Being". Asia-Pacific Psychiatry 11 (2019).

Volume 2 Issue 8 August 2019

(C) All rights are reserved by Uriel Halbreich. 